\title{
Gastric emptying is rapid in adolescents with type 1 diabetes and relates to gastrointestinal symptoms
}

\author{
Shiree Perano ${ }^{1,2^{*}}$, Christopher Rayner ${ }^{2,3}$, Stamatiki Kritas $^{4}$, Christine Mpundu-Kaambwa ${ }^{5}$, Kim Donaghue ${ }^{6}$, \\ Michael Horowitz ${ }^{2,7}$, Jennifer Couper ${ }^{1,2}$
}

From 8th APPES Biennial Scientific Meeting

Darwin, Australia. 29 October - 1 November 2014

Gastric emptying is a critical determinant in postprandial glycaemic control. This study aimed to assess whether gastric emptying in adolescents with type 1 diabetes (T1D) relates to gastrointestinal symptoms and to heart rate variability (HRV) as a measure of autonomic function.

We studied 30 adolescents (age $15 \pm 2.5$ years, BMI $22 \pm$ $3.1 \mathrm{~kg} / \mathrm{m}^{2}$ ) with T1D. Subjects consumed a ${ }^{13} \mathrm{C}$ labelled pancake meal. Gastric emptying was measured by ${ }^{13} \mathrm{C}$ breath test. Blood glucose was monitored frequently over 4 hours and gastrointestinal symptoms at 30-60 minute intervals, by a visual analogue questionnaire. Chronic gastrointestinal symptoms over the previous 3 months were assessed by a validated Diabetes Bowel Symptoms Questionnaire [1]. HRV was assessed by LabChart Pro [2].15 age and sex matched controls were also studied.

Gastric half emptying time was accelerated in adolescents with T1D compared to controls; 77.6 (61.4-99.3) minutes [median (IQR)] versus 109.1 (70.8-124.2), $\mathrm{P}=$ 0.02 ), independent of hyperglycaemia during the study, HbA1c, duration of diabetes, and BMI.

There was no difference in the prevalence of chronic symptoms or symptoms of a severity that affected lifestyle between the two groups.

The presence of nausea, vomiting, bloating and/or fullness during the study in T1D was associated with faster gastric emptying compared to asymptomatic T1D $(\mathrm{r}=0.55 ; \mathrm{p}=0.04)$, and this was independent of peak glucose and glucose at 4 hours.

Rate of gastric emptying in T1D did not correlate with HRV.
Adolescents with T1D have rapid gastric emptying associated with acute gastrointestinal symptoms. Symptomatology could be used as a clinical tool to determine the need for further investigation.

\section{Authors' details}

'Diabetes and Endocrinology Department, Women's and Children's Hospital, North Adelaide, SA, Australia. 'University of Adelaide, Adelaide, SA, Australia.

${ }^{3}$ Gastroenterology Department, Royal Adelaide Hospital, SA, Australia. ${ }^{4}$ Gastroenterology Department, Women's and Children's Hospital, North Adelaide, SA, Australia. ${ }^{5}$ Research and Evaluation Unit, Women's and Children's Hospital, North Adelaide, SA, Australia. ${ }^{6}$ Institute of Endocrinology, Children's Hospital Westmead, Sydney, NSW, Australia. ${ }^{7}$ Endocrinology Department, Royal Adelaide Hospital, North Terrace, SA, Australia.

\section{Published: 28 April 2015}

\section{References}

1. Quan C, Talley NJ, Cross S, Jones M, Hammer J, Giles N, et al: Development and validation of the Diabetes Bowel Symptom Questionnaire. Aliment Pharmacol Ther 2003, 17(9):1179-1187.

2. Task Force of the European Society of Cardiology and the North American Society of Pacing and Electrophysiology:: Heart rate variability: standards of measurement, physiological interpretation and clinical use. . Circulation Circulation 1996, 93(5):1043-1065.

doi:10.1186/1687-9856-2015-S1-O46

Cite this article as: Perano et al:: Gastric emptying is rapid in adolescents with type 1 diabetes and relates to gastrointestinal symptoms. International Journal of Pediatric Endocrinology 20152015 (Suppl 1):046.

'Diabetes and Endocrinology Department, Women's and Children's Hospital, North Adelaide, SA, Australia

Full list of author information is available at the end of the article 